\title{
Steering laws and continuum models for planar formations
}

\author{
E. W. Justh \\ Institute for Systems Research \\ University of Maryland \\ College Park, MD 20742, USA \\ justheisr.umd.edu
}

\author{
P. S. Krishnaprasad \\ Institute for Systems Research and \\ Dept. of Electrical and Computer Engineering \\ University of Maryland \\ College Park, MD 20742, USA \\ krishna@isr.umd.edu
}

\begin{abstract}
We consider a Lie group formulation for the problem of control of formations. Vehicle trajectories are described using the planar Frenet-Serret equations of motion, which capture the evolution of both vehicle position and orientation for unit-speed motion subject to curvature (steering) control. The Lie group structure can be exploited to determine the set of all possible (relative) equilibria for arbitrary $G$-invariant curvature controls, where $G=S E(2)$ is a symmetry group for the control law. The main result is a convergence result for $n$ vehicles (for finite $n$ ), using a Lyapunov function which for $n=2$, has been previously shown to yield global convergence. A continuum formulation of the basic equations is also presented.
\end{abstract}

\section{INTRODUCTION}

We consider formations (or swarms) of vehicles which are modeled as point-particles with unit-speed motion, subject to steering (curvature) control. Our initial practical motivation for considering such systems was the problem of coordinating formations of meter-scale UAVs (unmanned aerial vehicles), but our results may have implications for other types of unmanned vehicles, as well as certain biological swarming or schooling systems.

In section II, we discuss a Lie group setting for the problem of control of formations. The setting emerges naturally from the analysis of basic cases and concomitant physical contraints on the controls. (A modern reference for control systems on Lie groups is Jurdjevic [1].) We review how the Lie-group structure can be exploited to determine the set of all possible (relative) equilibria for arbitrary $G$-invariant curvature controls, where $G=S E(2)$ is the symmetry group for the control law [2], [3]. Ideas of "shape control" on Lie groups arise in a natural way for this problem [4], [5], [6].

In section III, we present some control laws which appear, based on numerical simulation, to stabilize certain relative equilibria. The primary analytical result we present (in section IV) is a convergence result for formations of $n$ vehicles (for finite $n$ ), using a Lyapunov function which for $n=2$ has been shown in previous work to yield a global convergence result.

The use of curvature controls can be given the mechanical interpretation of steering unit-mass, unit-charge particles by magnetic fields (and hence by gyroscopic forces, which are associated with vector potentials). For a discussion of the general theory of such controls, see [7]. This is in contrast with other current approaches to formation control that are based on scalar potentials, both in the point particle [8] and rigid-body [9] setting. Furthermore, our emphasis on the Lie group structure of the control laws distinguishes our work from an established physics literature in the area of large collections of interacting particles subject to local interaction laws, and giving rise to pattern-forming systems, spatially localized coherent structures (e.g., flocks), and phase transitions (e.g., from disorder to order); see, for instance, [10], [11], [12], [13].

\section{DYNAMICS, SHAPE VARIABLES, AND RELATIVE EQUILIBRIA}

Consider $n$ vehicles modeled as point particles moving in the plane with unit speed. Figure 1 illustrates the trajectories of the vehicles, and their respective planar Frenet-Serret frames. Each Frenet-Serret frame consists of a unit tangent vector $\mathbf{x}_{j}$ and a unit normal vector $\mathbf{y}_{j}$ to the $j^{\text {th }}$ trajectory [14]. The control law specifies the steering commands $u_{1}, \ldots, u_{n}$ for each vehicle. The dynamical equations are thus

$$
\begin{aligned}
\dot{\mathbf{r}}_{j} & =\mathbf{x}_{j}, \\
\dot{\mathbf{x}}_{j} & =\mathbf{y}_{j} u_{j}, \\
\dot{\mathbf{y}}_{j} & =-\mathbf{x}_{j} u_{j},
\end{aligned}
$$

for $j=1, \ldots, n$. The overdot denotes differentiation with respect to time (where time is also the arclength parameter, due to the unit speed assumption). The dynamics can also be written in polar coordinates as

$$
\dot{\mathbf{r}}_{j}=\left(\begin{array}{c}
\cos \theta_{j} \\
\sin \theta_{j}
\end{array}\right), \quad \dot{\theta}_{j}=u_{j}, \quad j=1, \ldots, n .
$$

There is a natural Lie group structure evident in system (1). Specifically, if $g_{j}$ is represented as

$$
g_{j}=\left[\begin{array}{ccc}
\mathbf{x}_{j} & \mathbf{y}_{j} & \mathbf{r}_{j} \\
0 & 0 & 1
\end{array}\right], j=1, \ldots, n,
$$

then the (collision-free) configuration submanifold $M_{\text {config }}$ on which the dynamics evolve is given by

$$
\begin{gathered}
M_{\text {config }}=\{\left(g_{1}, g_{2}, \ldots, g_{n}\right) \in \overbrace{G \times G \times \ldots \times G}^{n \text { copies }} \mid \mathbf{r}_{k} \neq \mathbf{r}_{j}, \\
1 \leq j \neq k \leq n\}
\end{gathered}
$$




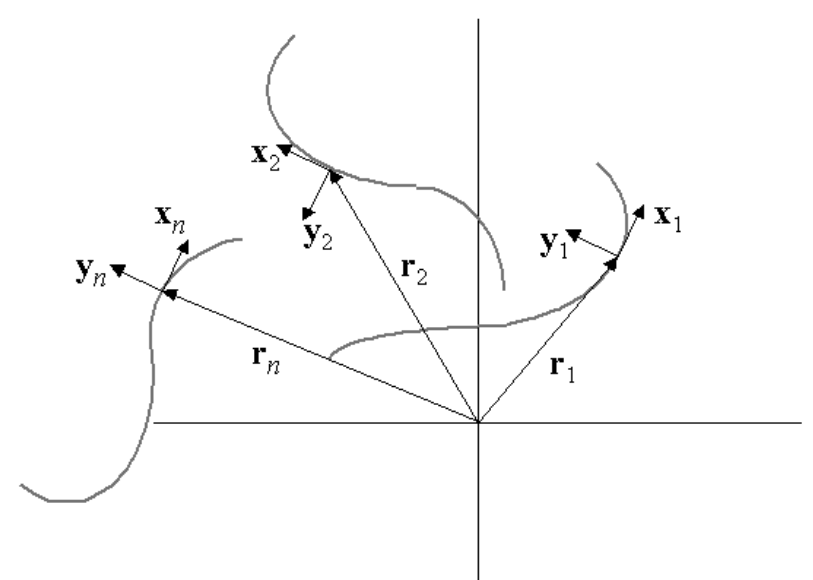

Fig. 1. Particle (i.e., vehicle) trajectories and their associated Frenet-Serret frames for unit-speed planar motion.

where $G=S E(2)$ is the special Euclidean group in the plane. The dynamics in configuration variables can be expressed as

$$
\dot{g}_{j}=g_{j} \xi_{j}, \quad j=1, \ldots, n
$$

where $\xi_{1}, \xi_{2}, \ldots \xi_{n} \in \mathfrak{g}$ (the Lie algebra of $G$ ), have the form

$$
\xi_{j}=A_{0}+A_{1} u_{j}, \forall j=1, \ldots, n,
$$

with

$$
A_{0}=\left[\begin{array}{lll}
0 & 0 & 1 \\
0 & 0 & 0 \\
0 & 0 & 0
\end{array}\right], \quad A_{1}=\left[\begin{array}{ccc}
0 & -1 & 0 \\
1 & 0 & 0 \\
0 & 0 & 0
\end{array}\right] \text {. }
$$

We define the shape variables

$$
\tilde{g}_{j}=g_{1}^{-1} g_{j}, \quad j=2, \ldots, n,
$$

which evolve on the reduced (shape) space

$$
\begin{aligned}
M_{\text {shape }}=\{ & \left(\tilde{g}_{2}, . ., \tilde{g}_{n}\right) \in \overbrace{G \times \ldots \times G}^{n-1} \mid\left(\tilde{g}_{j}\right)_{13}^{2}+\left(\tilde{g}_{j}\right)_{23}^{2}>0, \\
& \left.\left(\tilde{g}_{j}^{-1} \tilde{g}_{k}\right)_{13}^{2}+\left(\tilde{g}_{j}^{-1} \tilde{g}_{k}\right)_{23}^{2}>0,2 \leq j \neq k \leq n\right\},
\end{aligned}
$$

where $(\tilde{g})_{13}$ denotes the $(1,3)$-component of the matrix $\tilde{g}$, etc. (It is also possible to define shape variables in other combinations; e.g., $\tilde{g}_{j}=g_{j-1}^{-1} g_{j}, j=2, \ldots, n$ [5].) We consider control laws which have the property that the controls depend only on the shape variables; i.e., $\xi_{1}, \xi_{2}, \ldots, \xi_{n}$ depend only on the reduced variables $\tilde{g}_{2}, \tilde{g}_{3}, \ldots, \tilde{g}_{n}$. This leads to the following result (see [2], [3]):

Proposition 1: Consider the dynamics given by equations (5) and (6), evolving on the collision-free submanifold $M_{\text {config }}$ given by equation (4), where $G=S E(2)$ (and $\mathbf{r}_{j}$ is defined as in equation ( 3 ) for $j=1, \ldots, n$ ). Assume that the controls $u_{1}, u_{2}, \ldots, u_{n}$ depend only on the shape variables given by equation (8) (i.e., the controls are $G$-invariant).

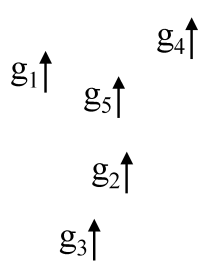

(a)

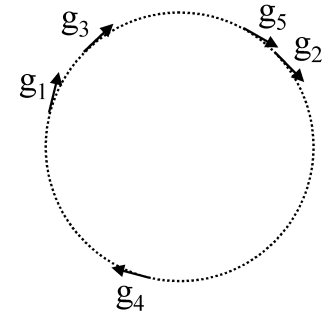

(b)
Fig. 2. Relative equilibria for the $n$-vehicle problem, illustrated for $n=5$ (arrows indicate tangent vectors to the vehicle trajectories): (a) for $u_{1}=$ $u_{2}=\ldots=u_{n}=0$, and (b) for $u_{1}=u_{2}=\ldots=u_{n} \neq 0$ [2], [3].

Then for equilibrium shapes (i.e., for relative equilibria of the dynamics (5) on configuration space), $u_{1}=u_{2}=\ldots=u_{n}$, and there are only two possibilities:

(a) $u_{1}=u_{2}=\ldots=u_{n}=0$, in which case a relative equilibrium consists of all vehicles heading in the same direction (with arbitrary relative positions within the formation), or

(b) $u_{1}=u_{2}=\ldots=u_{n} \neq 0$, in which case a relative equilibrium consists of all vehicles moving on the same circular orbit, with arbitrary chordal distances between them.

Proof: See [2], [3]

Figure 2 illustrates the two types of relative equilibria for the $n$-vehicle problem described in Proposition 1 [2], [3].

\section{FORMATION CONTROL LAWS}

Here we present two control laws which in simulation appear to stabilize the two types of relative equilibria. For one of the control laws, the rectilinear control law, we have previously proved a global convergence result for two vehicles [2], [3]. We explore the implications of this result for the $n$-vehicle problem (in section IV), and for a continuum setting for the problem of formation control (in section V).

\section{A. Rectilinear control law}

The rectilinear control law (i.e., the control law conjectured to stabilize certain relative equilibria with $u_{1}=\ldots=$ $u_{n}=0$ ) we have examined is given by equation (1) with

$$
\begin{aligned}
u_{j}=\frac{1}{n} \sum_{k \neq j}[ & -\eta\left(\frac{\mathbf{r}_{j k}}{\left|\mathbf{r}_{j k}\right|} \cdot \mathbf{x}_{j}\right)\left(\frac{\mathbf{r}_{j k}}{\left|\mathbf{r}_{j k}\right|} \cdot \mathbf{y}_{j}\right) \\
& \left.-f\left(\left|\mathbf{r}_{j k}\right|\right)\left(\frac{\mathbf{r}_{j k}}{\left|\mathbf{r}_{j k}\right|} \cdot \mathbf{y}_{j}\right)+\mu \mathbf{x}_{k} \cdot \mathbf{y}_{j}\right],
\end{aligned}
$$

where $\mathbf{r}_{j k}=\mathbf{r}_{j}-\mathbf{r}_{k}$. One possible choice for $f(\cdot)$ is

$$
f\left(\left|\mathbf{r}_{j k}\right|\right)=\alpha\left[1-\left(\frac{r_{o}}{\left|\mathbf{r}_{j k}\right|}\right)^{2}\right]
$$

where $r_{o}>0$, and where $\mu, \eta$, and $\alpha$ could be positive constants, or else functions of the inter-vehicle distance $\left|\mathbf{r}_{j k}\right|$. 


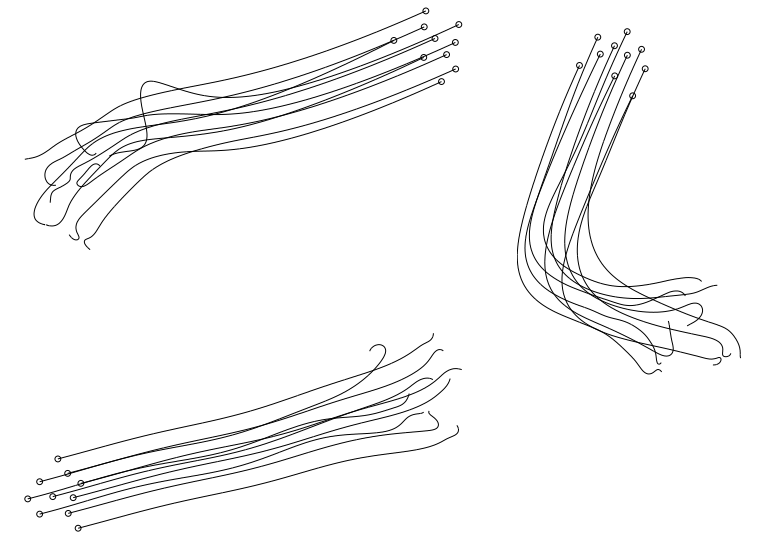

Fig. 3. Rectilinear formation initialization for ten vehicles from three different sets of random initial conditions [2], [3].

Figure 3 shows simulation results for the control law given by equation (10) [2], [3].

The Lyapunov function candidate we consider is

$$
V=\sum_{j=1}^{n} \sum_{k<j}\left[-\ln \left(\cos \left(\theta_{j}-\theta_{k}\right)+1\right)+h\left(\left|\mathbf{r}_{j}-\mathbf{r}_{k}\right|\right)\right],
$$

where we use the polar variables for ease of calculation, even though $V$ is $G$-invariant, and could be expressed in terms of the shape variables $\tilde{g}_{j}, j=2, \ldots, n$. For $n=2$, this Lyapunov function can be used to prove the following global convergence result [2], [3]:

Proposition 2: Consider the system given by equations (1) and (10) for $n=2$, with $\theta_{1}$ and $\theta_{2}$ defined by (2). Define $r=\left|\mathbf{r}_{2}-\mathbf{r}_{1}\right|$, and assume the following:

(A1) $\eta(\cdot), \mu(\cdot)$, and $f(\cdot)$ Lipschitz continuous on $(0, \infty)$;

(A2) $d h / d r=f(r)$, so that $h(\cdot)$ is continuously differentiable on $(0, \infty)$;

(A3) $\lim _{r \rightarrow 0} h(r)=\infty, \lim _{r \rightarrow \infty} h(r)=\infty, \exists \tilde{r}$ such that $h(\tilde{r})=0$; (A4) $\eta(r)>0, \mu(r)>0$, and $\mu(r)>\frac{1}{2} \eta(r), \forall r \geq 0$.

Define the set

$$
\Lambda=\left\{\left(r, \theta_{1}, \theta_{2}\right) \mid \cos \left(\theta_{2}-\theta_{1}\right) \neq-1 \text { and } 0<r<\infty\right\} .
$$

Then any trajectory starting in $\Lambda \subset M_{\text {shape }}$ converges to the set of equilibrium points contained in $\Lambda$.

Proof: See [2], [3].

There is a simple physical interpretation for the control law given by equation (10). The terms involving $\mu$ serve to align the heading directions of the vehicles. The terms involving $f(\cdot)$ steer the vehicles toward each other (if they are too far apart), or away from each other (if they are too close together). The terms involving $\eta$ tend to align the vehicles

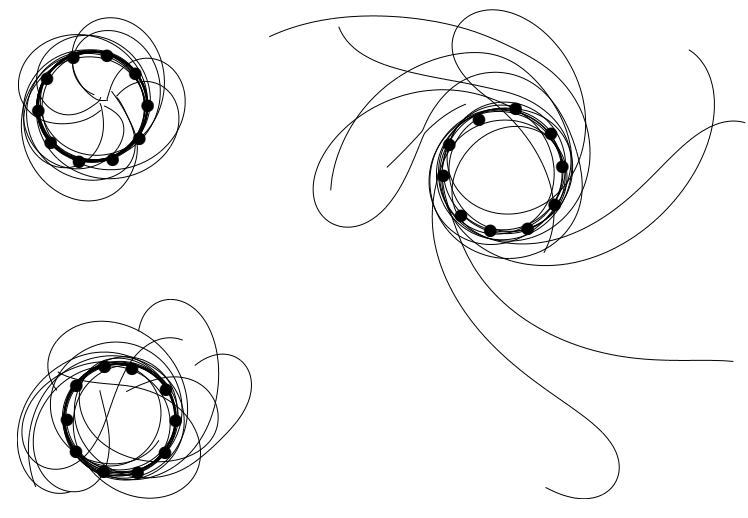

Fig. 4. Circling formation initialization for ten vehicles from three different sets of random initial conditions [3].

perpendicular to the baselines between the vehicles. Because of the summation, there is an averaging of the influences of all the other vehicles on the $j^{\text {th }}$ vehicle. This is consistent with many biological swarming and schooling models, which have (1) some mechanism for heading alignment, (2) repulsion or attraction based on separation distance or density, and (3) decreasing influence for neighbors at greater distances [15].

The inclusion of a collision-avoidance term, i.e., the term involving $f(\cdot)$ in our control laws, distinguishes our work from a model proposed by Vicsek, et. al. [10], and further studied (using graph-theoretic methods) by Jadbabaie, et. al. [16]. The Vicsek model is a discrete-time, unit-speed model in which each vehicle updates its heading direction at each time step by averaging its current heading direction with those of its neighbors located within a fixed distance of itself. (A noise term also contributes to the heading-direction updates.)

\section{B. Circling control law}

The circling control law (i.e., the control law conjectured to stabilize certain relative equilibria with $u_{1}=\ldots=u_{n} \neq 0$ ) we have examined is given by equation (1) with

$$
u_{j}=\frac{1}{n} \sum_{k \neq j}\left[\eta\left(\frac{\mathbf{r}_{j k}}{\left|\mathbf{r}_{j k}\right|} \cdot \mathbf{x}_{j}\right)-f\left(\left|\mathbf{r}_{j k}\right|\right)\left(\frac{\mathbf{r}_{j k}}{\left|\mathbf{r}_{j k}\right|} \cdot \mathbf{y}_{j}\right)\right],
$$

where, as for equation (10), $\mathbf{r}_{j k}=\mathbf{r}_{j}-\mathbf{r}_{k}$, and $f(\cdot)$ may be given by equation (11); but here $\eta$ may be either positive or negative.

Figure 4 shows simulation results for control law (14). Equation (14), like equation (10) for the rectilinear control law, can be expressed in terms of shape variables alone.

There is a simple physical interpretation for the circling control law (14), just as there was for the rectilinear control law. The terms involving $\eta$ tend to to align each vehicle perpendicular to the baselines between the vehicles, but in addition, each vehicle tries to keep the others to its left or right, depending on the sign of $\eta$. As in the rectilinear 
case, the terms involving $f(\cdot)$ serve to maintain appropriate intervehicle distances.

\section{STABILIZING CONTROL LAWS FOR $\boldsymbol{n}$ VEHICLES}

Ideally, we would like to prove convergence results for control laws (10) and (14), which based on numerical work, appear to have favorable convergence properties. As a first step in that direction, we address the question of whether a control law exists such that $V$ given by equation (12) can serve as a Lyapunov function for an $n$-vehicle system.

The derivative of $V$ along trajectories of equation (2), under assumption (A2), is

$$
\begin{aligned}
\dot{V}=\sum_{j=1}^{n} \sum_{k<j}\left[\left(\frac{\sin \left(\theta_{j}-\theta_{k}\right)}{\cos \left(\theta_{j}-\theta_{k}\right)+1}\right)\left(\dot{\theta}_{j}-\dot{\theta}_{k}\right)\right. \\
\left.+f\left(\left|\mathbf{r}_{j}-\mathbf{r}_{k}\right|\right) \frac{\left(\mathbf{r}_{j}-\mathbf{r}_{k}\right)}{\left|\mathbf{r}_{j}-\mathbf{r}_{k}\right|} \cdot\left(\dot{\mathbf{r}}_{j}-\dot{\mathbf{r}}_{k}\right)\right] \\
=\sum_{j=1}^{n} \sum_{k<j}\left[\left(\frac{\sin \left(\theta_{j}-\theta_{k}\right)}{\cos \left(\theta_{j}-\theta_{k}\right)+1}\right)\left(u_{j}-u_{k}\right)\right. \\
\left.\quad+f\left(\left|\mathbf{r}_{j}-\mathbf{r}_{k}\right|\right) \frac{\left(\mathbf{r}_{j}-\mathbf{r}_{k}\right)}{\left|\mathbf{r}_{j}-\mathbf{r}_{k}\right|} \cdot\left(\begin{array}{c}
\cos \theta_{j}-\cos \theta_{k} \\
\sin \theta_{j}-\sin \theta_{k}
\end{array}\right)\right] \\
=\sum_{j=1}^{n} \sum_{k<j}\left(\frac{\sin \left(\theta_{j}-\theta_{k}\right)}{\cos \left(\theta_{j}-\theta_{k}\right)+1}\right) \\
\times\left[\left(u_{j}-u_{k}\right)+f\left(\left|\mathbf{r}_{j}-\mathbf{r}_{k}\right|\right) \frac{\left(\mathbf{r}_{j}-\mathbf{r}_{k}\right)}{\left|\mathbf{r}_{j}-\mathbf{r}_{k}\right|} \cdot\left(\begin{array}{c}
\left.\sin \theta_{j}+\sin \theta_{k}\right) \\
\cos \theta_{j}+\cos \theta_{k}
\end{array}\right)\right] \\
=\sum_{j=1}^{n} \sum_{k \neq j}\left(\frac{\sin \left(\theta_{j}-\theta_{k}\right)}{\cos \left(\theta_{j}-\theta_{k}\right)+1}\right) \\
\times
\end{aligned}
$$

provided $\cos \left(\theta_{j}-\theta_{k}\right) \neq-1$, and where we have used the following identity:

$$
\begin{aligned}
\left(\frac{\sin \left(\theta_{j}-\theta_{k}\right)}{\cos \left(\theta_{j}-\theta_{k}\right)+1}\right)\left(\begin{array}{c}
-\left(\sin \theta_{j}+\sin \theta_{k}\right) \\
\cos \theta_{j}+\cos \theta_{k}
\end{array}\right) \\
-\left(\begin{array}{c}
\cos \theta_{j}-\cos \theta_{k} \\
\sin \theta_{j}-\sin \theta_{k}
\end{array}\right)=0 .
\end{aligned}
$$

Proposition 3: Consider the system given by equations (5) and (6), evolving on the configuration submanifold $M_{\text {config }}$ given by equation (4). Corresponding to this system, there is a reduced system evolving on the shape submanifold $M_{\text {shape }}$ given by equation (9). Let $V$ be given by equation (12), and assume that (A2) and (A3) hold, with $f(\cdot)$ Lipschitz continuous. Then there exist bounded controls $u_{1}, \ldots, u_{n}$, expressible as feedback functions of the shape variables, and a corresponding positively invariant set $\Omega \subset M_{\text {shape }}$ of the reduced dynamics which is a compact sublevel set of $V$. Furthermore, all trajectories starting in $\Omega$ converge to the largest invariant set $M$ of the set $E$ of points in $\Omega$ where
$\dot{V}=0$, and $M$ contains an equilibrium of the reduced system (corresponding to $\theta_{j}-\theta_{k}=0, \forall j, k$ ).

Proof: By the hypotheses on $h(\cdot)$, it follows that $\sum_{j=1}^{n} \sum_{k<j} h\left(\left|\mathbf{r}_{j}-\mathbf{r}_{k}\right|\right)$ assumes a minimum value on $M_{\text {shape }}$. Without loss of generality (since we can add an arbitrary constant to $V$ ), we may assume that this minimum value is zero. It follows that the minimum value $V_{\min }$ of $V$ is

$$
V_{\text {min }}=-\sum_{j=1}^{n} \sum_{k<j} \ln (2)=-\frac{n(n-1)}{2} \ln (2) .
$$

We define

$$
V_{\text {max }}=V_{\text {min }}+\ln (2)-\ln (1)=-\left(\frac{n(n-1)}{2}-1\right) \ln (2),
$$

and

$$
\Omega=\left\{\left(\tilde{g}_{2}, \ldots, \tilde{g}_{n}\right) \mid V\left(\tilde{g}_{2}, \ldots, \tilde{g}_{n}\right) \leq V_{\max }\right\},
$$

and we note that the radial unboundedness of $V$, along with the existence of $V_{\min }<V_{\max }$, implies that $\Omega$ is compact. Then for $\left(\tilde{g}_{2}, \ldots, \tilde{g}_{n}\right) \in \Omega$, we have $\left|\theta_{j}-\theta_{k}\right| \leq \pi / 2, \forall j, k$ (because otherwise $V$ would necessarily exceed $V_{\max }$ ).

Now we turn to the issue of choosing $u_{j}$ so that $\dot{V} \leq 0$. We seek $u_{j}$ such that $\dot{V}$ takes the form

$$
\dot{V}=-\sum_{j=1}^{n} \sum_{k \neq j}\left(\frac{\sin \left(\theta_{j}-\theta_{k}\right)}{\cos \left(\theta_{j}-\theta_{k}\right)+1}\right) p\left(\theta_{j}, \theta_{k}\right),
$$

where $p\left(\theta_{j}, \theta_{k}\right)$ is a bounded function of the shape variables which vanishes at equilibrium shapes, and results in $\dot{V} \leq 0$. (An example would be $p\left(\theta_{j}, \theta_{k}\right)=\sin \left(\theta_{j}-\theta_{k}\right)$.) Equating equations (15) and (20) gives

$$
\begin{aligned}
\sum_{j=1}^{n} \sum_{k \neq j} & \left(\frac{\sin \left(\theta_{j}-\theta_{k}\right)}{\cos \left(\theta_{j}-\theta_{k}\right)+1}\right) u_{j} \\
=- & \sum_{j=1}^{n} \sum_{k \neq j}\left(\frac{\sin \left(\theta_{j}-\theta_{k}\right)}{\cos \left(\theta_{j}-\theta_{k}\right)+1}\right) \\
& \times\left[p\left(\theta_{j}, \theta_{k}\right)+f\left(\left|\mathbf{r}_{j}-\mathbf{r}_{k}\right|\right) \frac{\left(\mathbf{r}_{j}-\mathbf{r}_{k}\right)}{\left|\mathbf{r}_{j}-\mathbf{r}_{k}\right|} \cdot\left(\begin{array}{c}
-\sin \theta_{j} \\
\cos \theta_{j}
\end{array}\right)\right] .
\end{aligned}
$$

The solution to equation (21) which minimizes $\sum_{j=1}^{n} u_{j}^{2}$ is

$$
u_{j}=\frac{-\left[\sum_{k \neq j}\left(\frac{\sin \left(\theta_{j}-\theta_{k}\right)}{\cos \left(\theta_{j}-\theta_{k}\right)+1}\right)\right]\left[\sum_{l=1}^{n} \sum_{k \neq l}\left(\frac{\sin \left(\theta_{l}-\theta_{k}\right)}{\cos \left(\theta_{l}-\theta_{k}\right)+1}\right) q_{l k}\right]}{\sum_{l=1}^{n}\left(\sum_{k \neq l} \frac{\sin \left(\theta_{l}-\theta_{k}\right)}{\cos \left(\theta_{l}-\theta_{k}\right)+1}\right)^{2}},
$$

where

$$
q_{j k}=p\left(\theta_{j}, \theta_{k}\right)+f\left(\left|\mathbf{r}_{j}-\mathbf{r}_{k}\right|\right) \frac{\left(\mathbf{r}_{j}-\mathbf{r}_{k}\right)}{\left|\mathbf{r}_{j}-\mathbf{r}_{k}\right|} \cdot\left(\begin{array}{c}
-\sin \theta_{j} \\
\cos \theta_{j}
\end{array}\right) .
$$




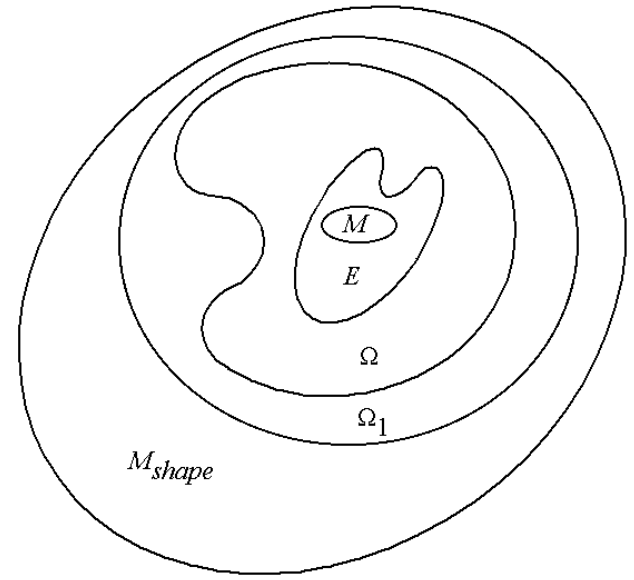

Fig. 5. Sets defined in Proposition 3: $M_{\text {shape }}$ is the manifold on which the shape variables evolve; $\Omega$ is the sublevel set of $V$ containing the initial shape; $\Omega_{1}$ is the set (containing $\Omega$ ) on which condition (25) is satisfied; $E$ is the set of points in $\Omega$ where $\dot{V}=0$; and $M$ is the largest invariant set contained in $E$.

However, a necessary condition for equation (22) to be meaningful is

$$
\sum_{j=1}^{n}\left(\sum_{k \neq j} \frac{\sin \left(\theta_{j}-\theta_{k}\right)}{\cos \left(\theta_{j}-\theta_{k}\right)+1}\right)^{2}>0
$$

It can be shown that inside $\Omega$, we have

$$
\frac{\sum_{j=1}^{n} \sum_{k \neq j}\left(\frac{\sin \left(\theta_{j}-\theta_{k}\right)}{\cos \left(\theta_{j}-\theta_{k}\right)+1}\right)^{2}}{\sum_{j=1}^{n}\left(\sum_{k \neq j} \frac{\sin \left(\theta_{j}-\theta_{k}\right)}{\cos \left(\theta_{j}-\theta_{k}\right)+1}\right)^{2}}<c<\infty,
$$

for some constant $c>0$.

The hypotheses on $h(\cdot)$, along with the boundedness assumption on $p(\cdot)$ and condition (25), ensure that the $u_{j}$ given by equation (22) exist and remain bounded on $\Omega$. (Furthermore, the $u_{j}$ are easily seen to be functions of shape variables alone.) Thus, on $\Omega$, trajectories of equations (5) and (6), with $u_{j}$ given by equation (22), exist and are unique. But we also have $\dot{V} \leq 0$ on $\Omega$, so $\Omega$ is a positively invariant set for the (shape) dynamics; i.e., trajectories which start in $\Omega$ remain in $\Omega$ for all future time. Then by LaSalle's Invariance Principle [17], trajectories which start in $\Omega$ converge to the largest invariant set $M$ contained in the set $E$ of points in $\Omega$ where $\dot{V}=0$ (see figure 5). $M$ includes at least one equilibrium shape with $\theta_{j}-\theta_{k}=0, \forall j, k$; namely, the shape for which $V=V_{\min }$ is attained.

\section{CONTINUUM FORMUlATION}

Here we introduce a continuum formulation appropriate for studying formation control of very large numbers of vehicles. This formulation naturally extends the approach we have taken above to the $n$-vehicle problem, and it incorporates the unit-speed assumption. The resulting continuum model evolves on the manifold $G=S E(2)$, a threedimensional manifold, rather than on $\mathbb{R}^{2}$, as do existing continuum models for planar formations found in the literature (c.f., [11]).

Instead of treating a very large number of vehicles as discrete point particles, we define a vehicle density function $\rho(t, \mathbf{r}, \theta)$, which describes the density of vehicles as a function of time $t$, position $\mathbf{r}$, and heading-angle $\theta$. The continuity equation (Liouville equation) is

$$
\frac{\partial \rho}{\partial t}=-\nabla \cdot(X \rho),
$$

where the vector field $X$ is given in coordinates as

$$
\left(\begin{array}{c}
d \mathbf{r} / d t \\
d \theta / d t
\end{array}\right)=\left(\begin{array}{c}
\cos \theta \\
\sin \theta \\
u
\end{array}\right)
$$

Thus, the continuity equation can be written in coordinates as

$$
\frac{\partial \rho}{\partial t}=-\left[\frac{\partial(u \rho)}{\partial \theta}+\left(\begin{array}{c}
\cos \theta \\
\sin \theta
\end{array}\right) \cdot \nabla_{\mathbf{r}} \rho\right] .
$$

Since "matter" is conserved, we may assume (or verify) that

$$
\int_{G} \rho(t, \mathbf{r}, \theta) d \mathbf{r} d \theta=1, \forall t .
$$

The energy functional analogous to equation (12) is

$$
\begin{aligned}
V_{c}(t)=\frac{1}{2} \int_{\Omega} \int_{\Omega}[- & \ln (\cos (\theta-\tilde{\theta})+1)+h(|\mathbf{r}-\tilde{\mathbf{r}}|)] \\
& \times \rho(t, \mathbf{r}, \theta) \rho(t, \tilde{\mathbf{r}}, \tilde{\theta}) d \mathbf{r} d \theta d \tilde{\mathbf{r}} d \tilde{\theta}
\end{aligned}
$$

where we assume that the support of $\rho(t, \mathbf{r}, \theta)$ lies in $\Omega \subset \Omega_{c}$, where

$\Omega_{c}=\left\{(\mathbf{r}, \theta) \in G \mid \cos \left(\theta-\theta_{o}\right) \geq \frac{\sqrt{2}}{2}, \forall \theta\right.$, for some $\left.\theta_{o}\right\}$,

and that $h(\cdot)$ is such that $V_{c}(t)$ is well-defined.

Formally differentiating equation (30) along trajectories of equation (28) (assuming, e.g., a compact region of integration $\Omega \subset \Omega_{c}$ with periodic boundary conditions) gives, in analogy to equation (15),

$$
\begin{array}{r}
\frac{d V}{d t}=\int_{\Omega} \int_{\Omega}[-\ln (\cos (\theta-\tilde{\theta})+1)+h(|\mathbf{r}-\tilde{\mathbf{r}}|)] \\
\times \frac{\partial \rho}{\partial t}(t, \mathbf{r}, \theta) \rho(t, \tilde{\mathbf{r}}, \tilde{\theta}) d \mathbf{r} d \theta d \tilde{\mathbf{r}} d \tilde{\theta} \\
=-\int_{\Omega} \int_{\Omega}[-\ln (\cos (\theta-\tilde{\theta})+1)+h(|\mathbf{r}-\tilde{\mathbf{r}}|)] \\
\quad \times\left[\frac{\partial(u \rho)}{\partial \theta}+\left(\begin{array}{c}
\cos \theta \\
\sin \theta
\end{array}\right) \cdot \nabla_{\mathbf{r}} \rho\right] \rho(t, \tilde{\mathbf{r}}, \tilde{\theta}) d \mathbf{r} d \theta d \tilde{\mathbf{r}} d \tilde{\theta} \\
=\int_{\Omega} \int_{\Omega}\left[\begin{array}{l}
u(t, \mathbf{r}, \theta) \frac{\partial}{\partial \theta}[-\ln (\cos (\theta-\tilde{\theta})+1)] \\
\left.+\left(\begin{array}{c}
\cos \theta \\
\sin \theta
\end{array}\right) \cdot \nabla_{\mathbf{r}} h(|\mathbf{r}-\tilde{\mathbf{r}}|)\right] \\
\times \rho(t, \mathbf{r}, \theta) \rho(t, \tilde{\mathbf{r}}, \tilde{\theta}) d \mathbf{r} d \theta d \tilde{\mathbf{r}} d \tilde{\theta}
\end{array}\right.
\end{array}
$$




$$
\begin{aligned}
=\int_{\Omega} \int_{\Omega} & \left(\frac{\sin (\theta-\tilde{\theta})}{\cos (\theta-\tilde{\theta})+1}\right) \\
\times & {\left[u(t, \mathbf{r}, \theta)+\left(\begin{array}{c}
-\sin \theta \\
\cos \theta
\end{array}\right) \cdot f(|\mathbf{r}-\tilde{\mathbf{r}}|) \frac{(\mathbf{r}-\tilde{\mathbf{r}})}{|\mathbf{r}-\tilde{\mathbf{r}}|}\right] } \\
& \times \rho(t, \mathbf{r}, \theta) \rho(t, \tilde{\mathbf{r}}, \tilde{\theta}) d \mathbf{r} d \theta d \tilde{\mathbf{r}} d \tilde{\theta}
\end{aligned}
$$

Similarly, we may write a formal expression for $u(t, \mathbf{r}, \theta)$ which is analogous to equation (22), or to equation (10) or (14).

One advantage of this type of continuum formulation is that it only involves two scalar fields: the density $\rho(t, \mathbf{r}, \theta)$, and the steering control $u(t, \mathbf{r}, \theta)$. The tradeoff is that the underlying space is three-dimensional instead of twodimensional (for planar formations), and there is a multiplicative nonlinearity in the Liouville equation (28); i.e., $u$ multiplies $\rho$.

\section{DIRECTIONS FOR FUTURE RESEARCH}

We have shown how a Lie group formulation provides insight into the problem of steering control for planar formations. Our main result, Proposition 3, shows that it is possible to prove convergence results for $n$ vehicles (although we have only proved global convergence for $n=2$ ). We have also shown how a continuum theory emerges naturally from the analysis of the $n$-vehicle problem. This work is still at an early stage, and there are many possible directions for future research, including (1) convergence analysis for the rectilinear (for $n>2$ ) and circling control laws presented in section III, (2) further study of the continuum model, e.g., by including temporal and spatial derivatives in the expression for the control field $u(t, \mathbf{r}, \theta)$, and (3) three-dimensional formation control.

\section{ACKNOWLEDGEMENTS}

This research was supported in part by the Naval Research Laboratory under Grants No. N00173-02-1G002 and N00173-03-1G001, by the Air Force Office of Scientific Research under AFOSR Grant No. F49620-01-0415, and by the Army Research Office under ODDR\&E MURI01 Program Grant No. DAAD19-01-1-0465 to the Center for Communicating Networked Control Systems (through Boston University).

\section{REFERENCES}

[1] V. Jurdjevic, Geometric Control Theory, Cambridge: Cambridge Univ. Press, 1997.

[2] E.W. Justh and P.S. Krishnaprasad, "A simple control law for UAV formation flying," Institute for Systems Research Technical Report TR 2002-38, 2002.

[3] E.W. Justh and P.S. Krishnaprasad, "Equilibria and steering laws for planar formations," Systems and Control Letters, under review, 2003.
[4] R.G. Littlejohn and M. Reinsch, "Gauge fields in the separation of rotations and internal motions in the $n$ body problem," Rev. Mod. Phys., Vol. 69, No. 1, pp. 213-275, 1997.

[5] P.S. Krishnaprasad and D.P. Tsakiris, "G-snakes: nonholonomic kinematic chains on Lie groups," Proc. IEEE Conf. Decision and Control, Vol. 3, pp. 2955-2960, 1994 (see also Institute for Systems Research technical report ISR TR 94-27, 1994).

[6] F. Zhang, M. Goldgeier, and P.S. Krishnaprasad, "Control of small formations using shape coordinates," Proc. IEEE Int. Conf. Robotics and Automation, to appear, 2003.

[7] L.-S. Wang and P.S. Krishnaprasad, "Gyroscopic control and stabilization," J. Nonlinear Science, Vol. 2, pp. 367-415, 1992.

[8] P. Ogren, E. Fiorelli, and N.E. Leonard, "Formations with a Mission: Stable Coordination of Vehicle Group Maneuvers," Proc. MTNS, 2002.

[9] T.R. Smith, H. Hanssmann, and N.E. Leonard, "Orientation control of multiple underwater vehicles with symmetry-breaking potentials," Proc. IEEE Conf. Decision and Control, Vol. 5, pp. 4598-4603, 2001.

[10] T. Vicsek, A. Czirók, E.B.-Jacob, I. Cohen, and O. Shochet, "Novel type of phase transitions in a system of self-driven particles," Phys. Rev. Lett., Vol 75, pp. 1226-1229, 1995.

[11] J. Toner and Y. Tu, "Flocks, herds, and schools: A quantitative theory of flocking," Phys. Rev. E, Vol. 58, No. 4, pp. 4828-4858, 1998.

[12] H. Levine, W.-J. Rappel, and I. Cohen, "Selforganization in systems of self-propelled particles," Phys. Rev. E, Vol. 63, No. 1, pp. 017101-1 to 017101-4, 2000.

[13] A. Czirók, M. Matsushita, and T. Vicsek, "Theory of periodic swarming of bacteria: Application to Proteus mirabilis," Phys. Rev. E, Vol 63, No. 3, pp. 031915-1 to $031915-11,2001$.

[14] W.M. Boothby, An introduction to differentiable manifolds and Riemannian geometry, 2nd ed. Orlando: Academic Press, 1986.

[15] D. Grünbaum, "Schooling as a strategy for taxis in a noisy environment," in Animal Groups in Three Dimensions, J.K. Parrish and W.M. Hamner, eds., Cambridge: Cambridge University Press, 1997.

[16] A. Jadbabaie, J. Lin, and A.S. Morse, "Coordination of groups of mobile autonomous agents using nearest neighbor rules," IEEE Transactions on Automatic Control, Vol. 48, No. 6, pp. 988-1001, 2003 (also in Proc. IEEE Conf. Decision and Control, Vol. 3, pp. 29532958, 2002).

[17] H. Khalil. Nonlinear Systems. New York: Macmillan Publishing Co., 1992. 